Supporting Information for

\title{
Characterization of the presence and function of platelet opioid receptors
}

Sarah M. Gruba, ${ }^{1,2}$ Danielle H. Francis, ${ }^{2}$ Audrey F. Meyer, ${ }^{2,3}$ Eleni Spanolios, ${ }^{2}$ Jiayi He, ${ }^{2}$ Ben M. Meyer, ${ }^{2}$ Donghyuk Kim, ${ }^{2}$ Kang Xiong-Hang, ${ }^{2}$ and Christy L. Haynes ${ }^{2 *}$

${ }^{1}$ Present address: Boston Scientific, 4100 Hamline Ave N., St. Paul, MN 55112

${ }^{2}$ Department of Chemistry, University of Minnesota, Minneapolis, MN 55455

${ }^{3}$ Present address: Boston Scientific, 3 Scimed Place, Maple Grove, MN 55311

*Corresponding Author E-mail: chaynes@umn.edu

Anti-א Antibody

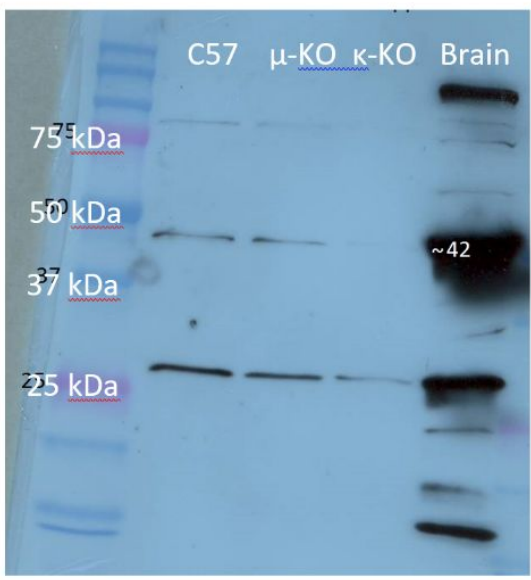

Anti- $\delta$ Antibody

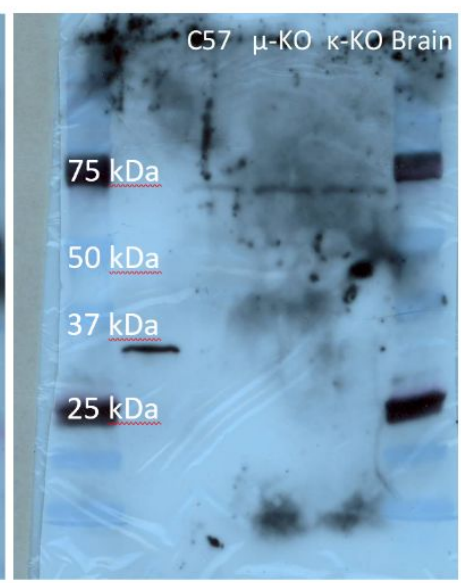

Anti- $\mu$ antibody

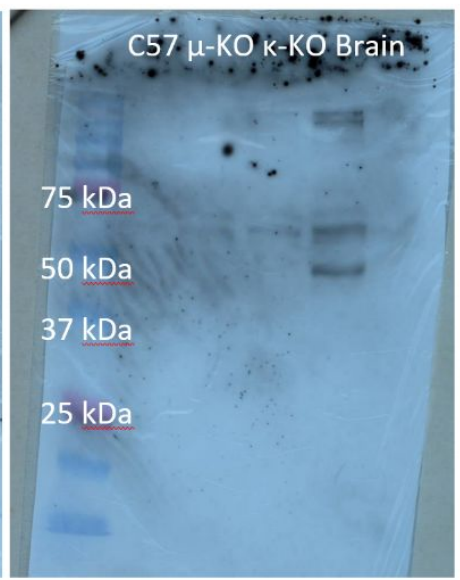

Figure SI 1: Western blots of knockout mouse platelets incubated with (A) anti- $\kappa,(B)$ anti- $\delta$, and (C) anti- $\mu$ antibodies. The anti- $\mu$ antibody used in the blots shown here was the one sourced from Millipore Sigma. The blot membrane was overlaid with the x-ray film before scanning. Numbers and labels were added to the images. 


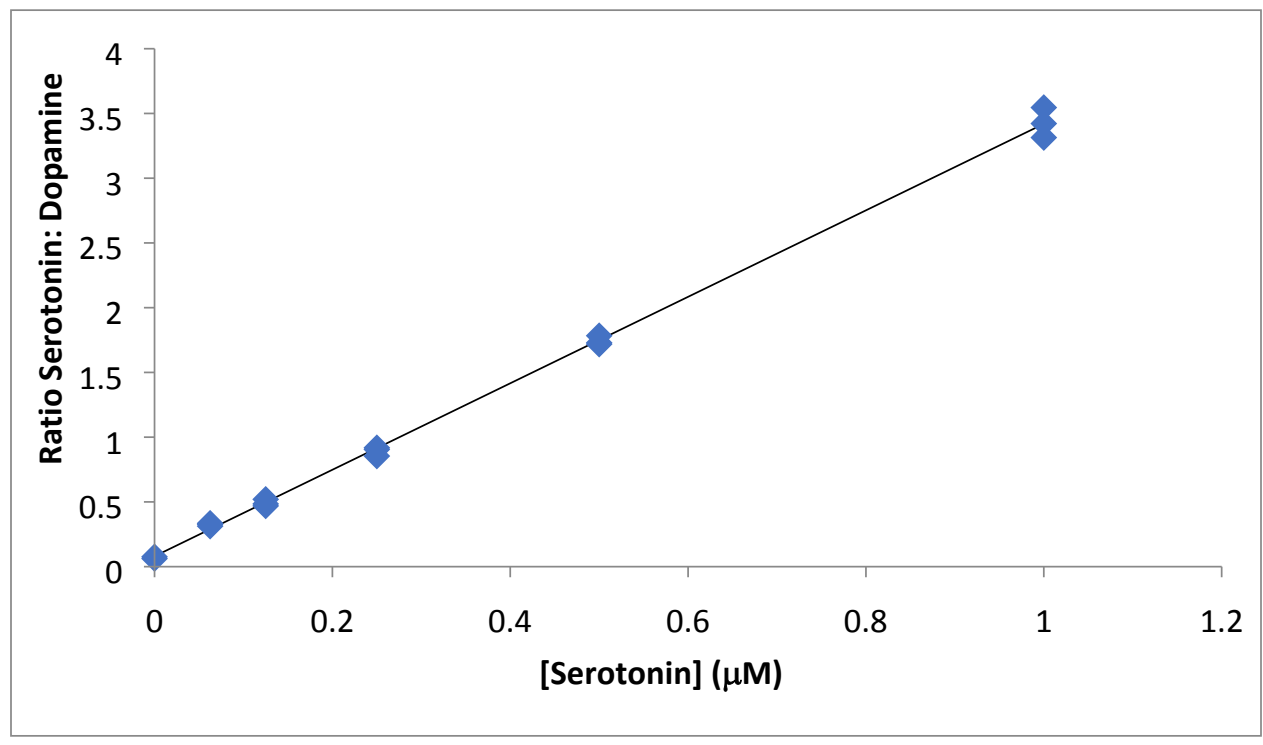

Figure SI 2. The calibration curve was constructed by plotting the ratio of the serotonin peak: dopamine peak (internal standard) from measured HPLC chromatograms. The serotonin calibration curve was made using three replicates of known serotonin concentrations $(0.0,0.0625,0.125,0.25,0.5$, and $1 \mu \mathrm{M})$. This calibration curve was used to calculate the limit of detection $(0.017 \mu \mathrm{M})$ and limit of quantification $(0.052$ $\mu \mathrm{M})$ for this method.

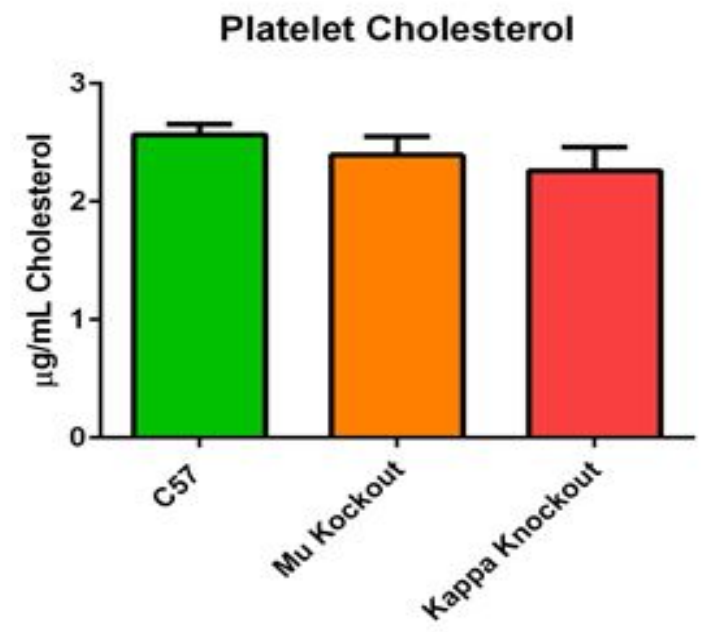

Figure SI 3: Comparison of cholesterol concentrations in platelets from control C57, $\mu-\mathrm{KO}$ and $\kappa-\mathrm{KO}$ mice. No statistical significance was noted. 


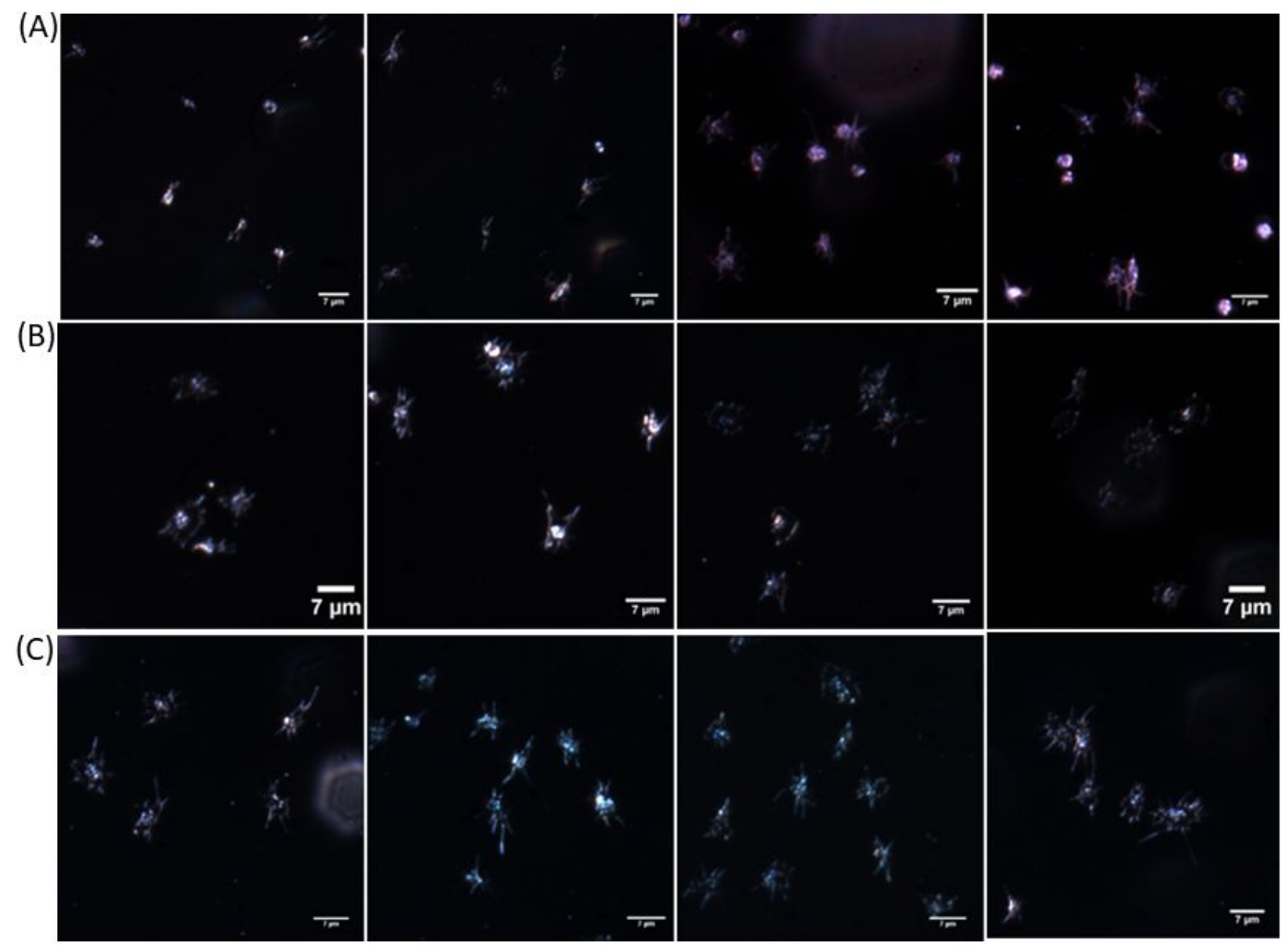

Figure SI 4: Dark-field images of representative activated (A) C-57 platelets, (B) $\kappa-K O$ platelets, and (C) $\mu$-KO platelets. The scale bar in all images represents $7 \mu \mathrm{m}$. 